\title{
Lesion-Induced Thalamocortical Axonal Plasticity in the S1 Cortex Is Independent of NMDA Receptor Function in Excitatory Cortical Neurons
}

\author{
Akash Datwani, ${ }^{1 *}$ Takuji Iwasato, ${ }^{2,3^{*}}$ Shigeyoshi Itohara, ${ }^{3}$ and Reha S. Erzurumlu ${ }^{1}$ \\ ${ }^{1}$ Department of Cell Biology and Anatomy and Neuroscience Center, Louisiana State University Health Sciences Center, \\ New Orleans, Louisiana 70112, ${ }^{2}$ PRESTO, Japan Science and Technology Corporation, and ${ }^{3}$ Laboratory for Behavioral \\ Genetics, Brain Science Institute, RIKEN, Saitama 351-0198, Japan
}

Neural activity plays an important role in refinement and plasticity of synaptic connections in developing vertebrate sensory systems. The rodent whisker-barrel pathway is an excellent model system to investigate the role of activity in formation of patterned neural connections and their plasticity. When whiskers on the snout or the sensory nerves innervating them are damaged during a critical period in development, whiskerspecific patterns are altered along the trigeminal pathway, including the primary somatosensory (S1) cortex. In this context, NMDA receptor (NMDAR)-mediated activity has been implicated in patterning and plasticity of somatosensory maps. Using CxNR1KO mice, in which NMDAR1 (NR1), the essential NMDAR subunit gene, is disrupted only in excitatory cortical neurons, we showed that NMDAR-mediated activity is essential for whisker-specific patterning of barrel cells in layer IV of the S1 cortex. In CxNR1KO mice, thalamocortical axons (TCAs)

The face representation area of the rodent primary somatosensory (S1) cortex is characterized by modular patterns of layer IV granule cells ("barrels") and thalamocortical axon (TCA) patches that fill these modules (Woolsey and Van der Loos, 1970; Killackey, 1973). The distribution of barrels replicates the spatial arrangement of the whiskers and sinus hairs on the contralateral snout. These patterns emerge sequentially along the somatosensory trigeminal neuraxis. They are called "barrelettes" in the brainstem trigeminal nuclei and "barreloids" in the ventroposteromedial (VPM) nucleus of the thalamus (Woolsey, 1990; Erzurumlu and Kind, 2001). The resulting array of presynaptic whisker-specific inputs and postsynaptic cellular modules confers neurons with preferential responsiveness to stimulation of individual whiskers. When nerve or whisker lesions perturb the sensory periphery during a critical period, there are corresponding

Received May 21, 2002; revised Aug. 8, 2002; accepted Aug. 16, 2002.

This work was supported by a grant-in-aid for Scientific Research from the Ministry of Education, Culture, Sports, Science, and Technology of Japan (T.I.) and by National Institutes of Health/National Institute of Neurological Disorders and Stroke Grant NS-39050 (R.S.E.). We thank R. Ando and Y. Taguchi for technical assistance.

*A.D and T.I. contributed equally to this work.

Correspondence should be addressed to either of the following: Dr. Reha S. Erzurumlu, Department of Cell Biology and Anatomy, Louisiana State University Health Sciences Center, 1901 Perdido Street, New Orleans, LA 70112, E-mail: rerzur@lsuhsc.edu; or Dr. Takuji Iwasato, Behavioral Genetics Laboratory, Brain Science Institute, The Institute of Physical and Chemical Research (RIKEN), 2-1, Hirosawa, Wako-shi, Saitama 351-0198, Japan, E-mail: iwasato@brain.riken.go.jp. Copyright $\odot 2002$ Society for Neuroscience $0270-6474 / 02 / 229171-05 \$ 15.00 / 0$ representing the large whiskers segregate into rudimentary patches, but barrels as cellular modules do not develop. In this study, we examined lesion-induced TCA plasticity in CxNR1KO mice. TCA patterns underwent normal structural plasticity when their peripheral inputs were altered after whisker lesions during the critical period. The extent of the lesion-induced morphological plasticity and the duration of the critical period were quantitatively indistinguishable between CxNR1KO and control mice. We conclude that TCA plasticity in the neocortex is independent of postsynaptic NMDAR activity in excitatory cortical neurons, and that non-NMDAR-mediated cortical activity and/or subcortical mechanisms must be operational in this process.

Key words: somatosensory cortex; thalamocortical synaptic plasticity; conditional knock-out; barrels; whiskers; glutamatergic transmission; Cre/loxP system

alterations in the physiological and morphological integrity of the barrel map. Specifically, if a row of whiskers is cauterized during the first few days after birth, representation of the deprived row shrinks, and that of neighboring intact barrel rows expands (for review, see Woolsey, 1990). The ratio of this "expansion/shrinkage" (i.e., structural plasticity) diminishes with age, and no obvious structural changes are evident when lesions are placed after postnatal day $3(\mathrm{P} 3)$.

Despite a wealth of information at the anatomic, physiological, and behavioral levels, the mechanisms underlying thalamocortical plasticity during development and at maturity have been elusive (Goodman and Shatz, 1993; Cramer and Sur, 1995; Katz and Shatz, 1996; Lebedev et al., 2000; Pallas, 2001; Wallace et al., 2001). Over the past decade, glutamatergic neural transmission and its regulation by serotonin $(5-\mathrm{HT})$ have received significant attention in the sculpting of connections in the barrel cortex. Several mutations in the genes encoding NMDA receptors (NMDARs), metabotropic glutamate receptors, 5-HT receptors, 5-HT transporters (5-HTTs), and altered levels of cortical 5-HT now reveal how presynaptic and postsynaptic whisker-specific patterns are established in the barrel cortex of mice (for review, see Erzurumlu and Kind, 2001).

One of these mutant mouse models (CxNR1KO mice) involves selective loss of functional NMDARs in cortical excitatory neurons (Iwasato et al., 2000). In these mice, the whiskers on the snout and all subcortical neural representations of the whiskers are intact. However, in the S1 cortex, layer IV granule cells fail to 
form barrels, although their presynaptic inputs from the VPM nucleus of the thalamus develop whisker-specific patterns that are less distinct than those seen in control littermates. Thus, the CxNR1KO mouse is an ideal genetic model to examine the role of cortical excitatory NMDAR function in plasticity of the TCA terminals during the critical period. We found that CxNR1KO mice have normal levels of lesion-induced TCA plasticity and duration of the critical period for this plasticity. Thus, NMDAR function in cortical excitatory neurons is not essential for the lesion-induced TCA plasticity.

\section{MATERIALS AND METHODS}

Experiments and analysis were performed in 208 neonatal mice in accordance with guidelines of the Institutional Animal Care and Use Committees of both of our institutes. Of these 208 neonates, 92 were CxNR1KO (Emx $\left.1^{\mathrm{Cre} /+} N R 1^{\text {flox }--}\right)$ mice, and 116 were littermate controls. The control mice we used were not wild-type mice but rather transgenic mice $\left(E m x 1^{+/+} N R 1^{\text {flox } /-}, E m x 1^{C r e /+} N R 1^{\text {flox } /+}\right.$, or $E m x 1^{+/+} N R 1^{\text {flox } /+}$ mice) derived from the crossing of $\mathrm{Emx} 1^{\mathrm{Cre} /+} \mathrm{NR} 1^{+/-}$or $\mathrm{Emx} 1^{\mathrm{Cre} / \mathrm{Cre}} \mathrm{NR} 1^{+/-}$mice with $N R 1^{\text {flox/flox }}$ mice as described previously (Iwasato et al., 2000). For each age group, 10-59 mice were used. P0-P6 neonatal mice were anesthetized by hypothermia, the center row (row C) whisker follicles were electrocauterized (Fig. 1 $A$ ), and damaged follicles were removed with forceps. The pups were allowed to recover and returned to their home cages. Between P7 and P9, the pups were killed by intraperitoneal overdose injection of sodium pentobarbital. The effectiveness of row $\mathrm{C}$ lesions was verified by two independent techniques: hematoxylin-eosin (HE) staining of $50 \mu \mathrm{m}$ tangential sections through the whisker pads and cytochrome oxidase (CO) histochemistry on coronal sections through brainstem trigeminal nuclei and the VPM nucleus of the thalamus.

For 5-HTT immunohistochemistry, $60 \mu \mathrm{m}$ tangential sections of the cerebral cortex were incubated in anti-5-HTT rabbit polyclonal antibody (1:10,000; DiaSorin, Stillwater, MN) overnight in PBS with $0.3 \%$ Triton $\mathrm{X}-100$. Sections were rinsed in PBS and then incubated in a secondary biotinylated goat anti-rabbit antibody (1:200; Sigma, St. Louis, MO), followed by processing with ABC (Vector Laboratories; Burlingame, CA) and DAB (Sigma) for light microscopy visualization. Sections were mounted on gelatin-coated slides, dehydrated, cleared in xylene, and coverslipped in DePeX mounting medium (BDH Laboratory Supplies, Poole, UK). In some instances, 5-HTT-immunostained sections were counterstained with cresyl violet (Nissl stain). For quantitative analysis, 5-HTT-immunostained tangential cortex sections were visualized under the light microscope, and images of the barrel field were acquired by a CoolSnap digital camera (Media Cybernetics, Carlsbad, CA). Measurements of the entire large whisker-representation areas (LWAs) and surface areas devoted to each 5-HTT-immunopositive barrel row (rows B-D) within the LWA were made using the Metaview Image Analysis Program (Universal Imaging Corp, Downingtown, PA). The barrel septa were included in the row area measurements, because these areas are also susceptible to encroachment by the TCAs from intact neighboring rows. We adopted the measurement scheme referred to as the $\mathrm{D} / \mathrm{C}$ ratio that was used previously (Schlaggar et al., 1993). For each hemisphere, the areas of rows B-D were normalized for the LWA and expressed as a percentage. Subsequently, the $\mathrm{D} / \mathrm{C}$ ratio was calculated by dividing the normalized row $\mathrm{D}$ percentage by the normalized row $\mathrm{C}$ percentage for control and $\mathrm{CxNR} 1 \mathrm{KO}$ cortices. The $\mathrm{D} / \mathrm{C}$ ratio provides a numerical value to the relative cortical territory devoted to rows $\mathrm{D}$ and $\mathrm{C}$ and the relative expansion in row $\mathrm{D}$ and reduction of row $\mathrm{C}$ in each hemisphere after peripheral lesion. The standardization corrects for flattening artifacts, normalizes for overall LWA, and provides a numerical index for the plasticity in both deprived and nondeprived rows. Consequently, one can effectively examine the degree of variation of plasticity after row $\mathrm{C}$ vibrissa follicle cauterization across different ages in control and CXNR1KO barrel cortices.

\section{RESULTS}

Electrocautery was performed on the center-row (row C) whisker follicles of CxNR1KO mice and littermate controls $\left(E m x 1^{+/+}\right.$ $N R 1^{\text {flox } /-}, E m x 1^{C r e /+} N R 1^{\text {flox } /+}$, or $E m x 1^{+/+} N R 1^{\text {flox } /+}$ ) between $\mathrm{P} 0$ and $\mathrm{P} 6$. Pups were killed by intraperitoneal overdose injection of sodium pentobarbital between P7 and P9, and the effects of
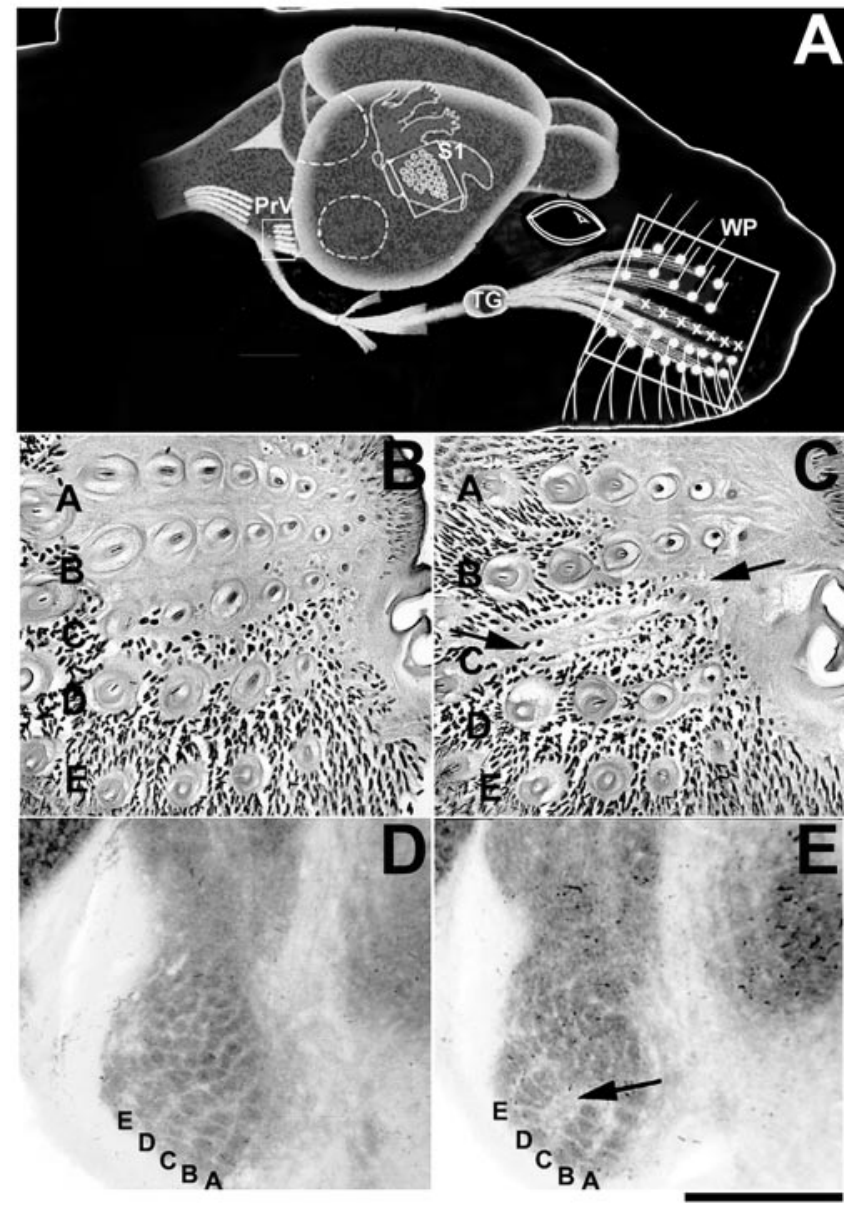

Figure 1. Peripheral and central effects of row C whisker lesions. $A$, Diagram of the whisker-barrel pathway illustrating row $\mathrm{C}$ lesion in the whisker pad $(W P)$ and effects in the principal sensory nucleus $(\operatorname{Pr} V)$ and the S1 cortex. $T G$, Trigeminal ganglion. $B, C$, HE-stained tangential sections through the whisker pad of a normal mouse $(B)$ and a row $C$ follicle electrocauterized control mouse $(C)$. Arrows indicate damaged row $\mathrm{C}$ whisker follicles, whereas other rows are uncompromised. $D, E, \mathrm{CO}$ histochemistry reveals normal representation of whiskers and whisker rows (i.e., barrelettes) in the principal sensory nucleus $(D)$ and the absence of row $\mathrm{C}$ representation after electrocautery $(E)$. Lesions were placed at P1, and the whisker pads and brainstem were examined at P8. Scale bar: $B, C, 200 \mu \mathrm{m} ; D, E, 500 \mu \mathrm{m}$.

electrocautery were clearly identified in tangential sections through the whisker pad (Fig. 1, compare $B$ with $C$ ).

Mitochondrial enzyme $\mathrm{CO}$ is used routinely as a histochemical marker for whisker-specific patterns along the rodent trigeminal pathway. We confirmed the whisker lesion effects in the brainstem trigeminal nuclei (Fig. 1D,E) and in the VPM nucleus of the thalamus (data not shown). In CxNR1KO and control mice, clear effects of row $\mathrm{C}$ lesions could be detected in the brainstem and thalamus when lesions were placed during the first $3 \mathrm{~d}$ after birth. In the principal sensory nucleus of the trigeminal nerve, $\mathrm{CO}$ patches corresponding to the row $\mathrm{C}$ whiskers were absent (Fig. $1 E$, arrow), and in the VPM nucleus, these patches fused into a narrow band (data not shown), as has been reported previously by others (for review, see Woolsey, 1990).

We visualized TCA patterns in layer IV of flattened cortices by immunohistochemistry for 5-HTT. The expression and localization of 5-HTT has been studied extensively in the developing rodent brain. Developing primary sensory neurons of the somato- 
sensory, auditory, and visual relay nuclei of the dorsal thalamus express the 5-HTT gene, and their cortical projections can be identified reliably by 5 -HTT immunohistochemistry (Lebrand et al., 1996, 1998). We found that in both CxNR1KO and control mice, TCAs corresponding to the damaged row $\mathrm{C}$ whiskers fused into a band. TCA patches corresponding to the neighboring row $\mathrm{B}$ and row $\mathrm{D}$ whiskers enlarged when row $\mathrm{C}$ whisker follicles were cauterized during the critical period that lasts through P3 (Fig. 2). Analysis of row $\mathrm{C}$ and row D areas normalized to LWA by means of ANOVA show that at all ages examined, the control row areas are always greater than corresponding row areas of $\mathrm{CxNR} 1 \mathrm{KO}$ (Fig. 3A,B) $(p<0.05)$. These results are consistent with our previous report that in the absence of functional NMDARs in excitatory cortical neurons, the amount of territory occupied by each TCA terminal patch decreases (Iwasato et al., 2000). Yet the overall shapes of the curves are similar, indicating that the relative reductions and expansions of row $\mathrm{C}$ (Fig. $3 A$ ) and row D (Fig. $3 B$ ), respectively, over various ages examined are comparable between CxNR1KO and control mice.

The effects of row $\mathrm{C}$ whisker lesions on the $\mathrm{D} / \mathrm{C}$ ratio at different postnatal days in control and $\mathrm{CxNR} 1 \mathrm{KO} \mathrm{S} 1$ cortex were not significantly different (Fig. 3C). After lesions at progressively older ages, the overall $\mathrm{D} / \mathrm{C}$ index of plasticity was diminished comparably for both groups. Qualitatively, we could discern slightly more plastic changes in the CxNR1KO animals at P3 (Fig. 2); however, this was not statistically significant when quantitative areal measurement and statistical analyses were performed. In some cases, 5-HTT immunostained sections were counterstained with Nissl stain to reveal cellular organization in barrel cortex (data not shown). As expected, there were no cellular patterns in the barrel cortex of experimental (with whisker lesions) or CxNR1KO animals without whisker lesions, indicating that even in the absence of postsynaptic cellular patterning, TCAs can undergo dramatic reorganization. This is significant because it has been suggested that the modular organization of layer IV barrel cells and expression of high levels of extracellular matrix proteins such as tenascin in barrel septa play a role in "cordoning off" TCA terminals and maintaining their patterned organization (Cooper and Steindler, 1986; Steindler et al., 1990; but see Jhaveri et al., 1991). Our results document that the whisker-specific pattern information is still relayed to the neocortex and maintained by the TCAs, even when there are no barrels or barrel boundaries in the neocortex and extracellular matrix distribution is uniform (Iwasato et al., 2000). In addition, structural plasticity of TCAs occurs regardless of patterning of postsynaptic elements and NMDAR function in excitatory cortical neurons.

\section{DISCUSSION}

Our results show that TCA plasticity and the extent of the critical period for this plasticity in the $\mathrm{S} 1$ cortex of $\mathrm{CxNR} 1 \mathrm{KO}$ mice are similar to control transgenic and normal mice. Thus, it is apparent that NMDAR function in the cortical excitatory neurons is not essential for the TCA plasticity. These results are in contrast to those of a previous pharmacological blockade study (Schlaggar et al., 1993), in which subdural application of Elvax implants loaded with the NMDAR antagonist APV over the postnatal rat S1 cortex blocked row $\mathrm{C}$ lesion-induced TCA plasticity. A number of possibilities may account for these different results. Interference with non-NMDA glutamatergic receptor systems of the APV doses used, action on the presynaptic thalamic neurons, and action on cortical neurons are among these possibilities. A later study by the same group showed significant alterations in single-

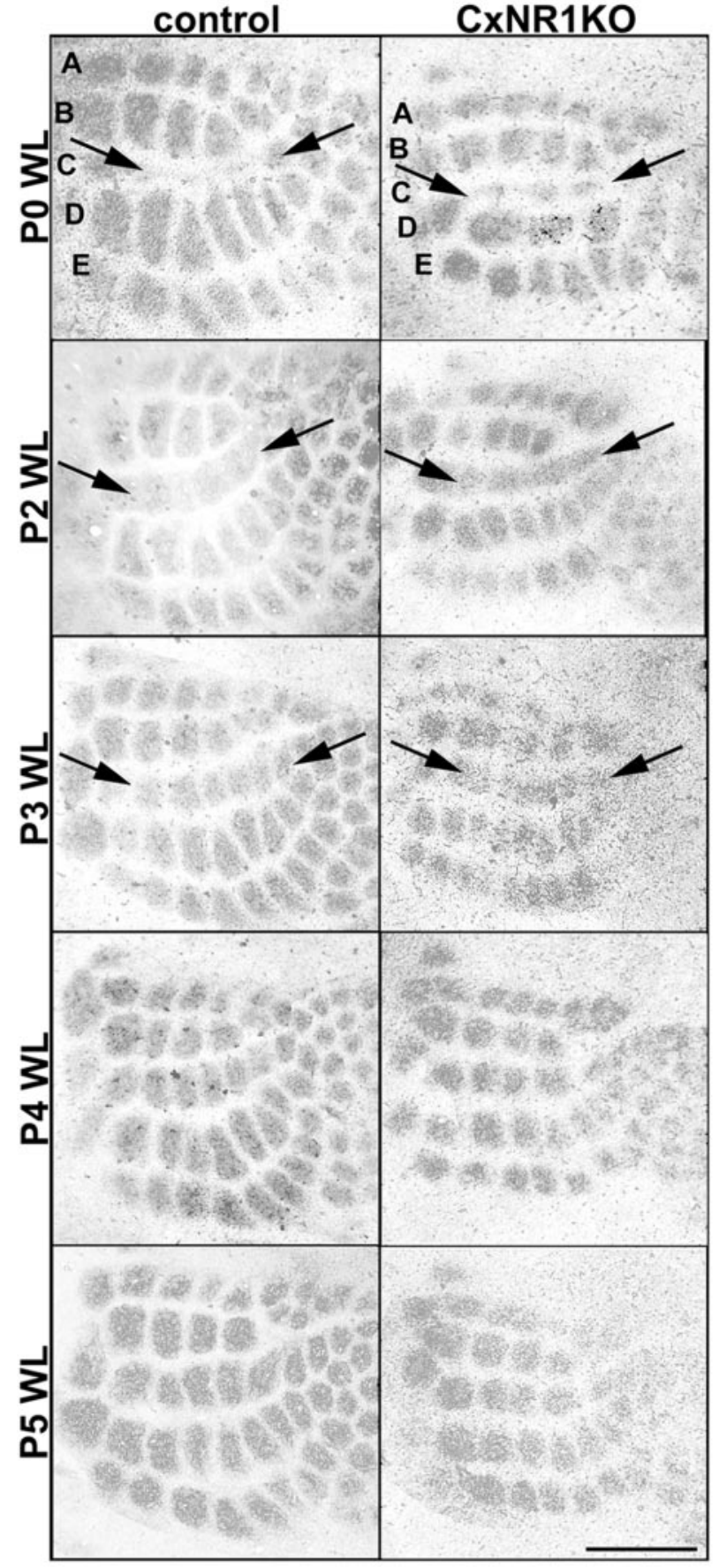

Figure 2. 5-HTT immunoreacted tangential sections through layer IV of the S1 cortex in both control and CxNR1KO pups demonstrate normal critical-period plasticity. Whisker lesions $(W L)$ at $\mathrm{P} 0$ resulted in robust alterations of TCAs in both control and CxNR1KO cases. Damaged row $\mathrm{C}$ representation areas were reduced dramatically, and flanking rows $\mathrm{B}$ and D areas were expanded. Lesions placed at subsequent ages P2 and P3 resulted in progressively less dramatic alterations in TCAs. Arrows indicate compromised row $\mathrm{C}$ as a fused band of TCAs in both control and CxNR1KO barrel cortex. Lesions placed at P4 or later were ineffective in imparting plastic changes in TCA patterning in both control and CxNR1KO mice. Scale bar, $500 \mu \mathrm{m}$.

whisker responsiveness of barrel neurons and functional organization of cortical columns in postnatal rats treated with APVloaded Elvax implants over the barrel cortex (Fox et al., 1996). The results of this electrophysiological study are in agreement 


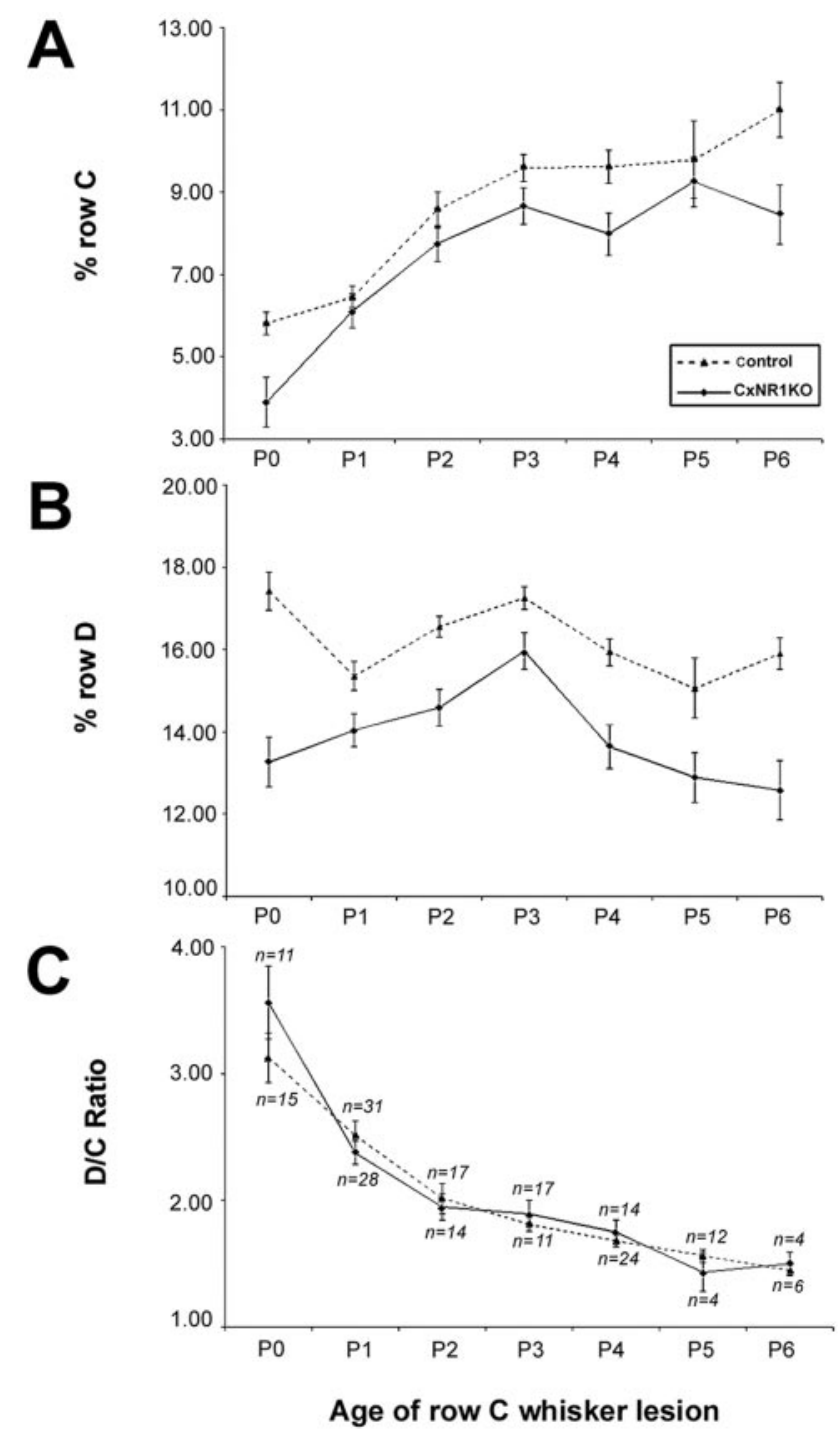

Figure 3. Quantification of lesion-induced critical-period plasticity in control and CxNR1KO barrel cortex. $A, B$, Analyses of normalized percentage row $\mathrm{C}$ and percentage row $\mathrm{D}$ areas at various ages of whisker lesions indicate that, despite the reduced areas of overall barrel rows in CxNR1KO S1 cortex (solid line), the shapes of the curves are similar, indicating that the TCAs respond similarly to the effects of peripheral lesions. $C$, The $\mathrm{D} / \mathrm{C}$ ratios are indistinguishable for both genotypes and at different ages of row $\mathrm{C}$ lesion and follow a graded diminution until $\mathrm{P} 3$. Lesions placed after P3 are not effective in reorganizing TCAs, because the critical period of barrel plasticity has lapsed. $n$ indicates number of animals. Error bars indicate SEM. Comparisons were made using ANOVA. For D/C ratios, there were no significant differences across all ages. Analysis of row D revealed significant differences between control (dashed line) and $\mathrm{CxNR} 1 \mathrm{KO}$ cortices at all ages: $\mathrm{P} 0, p<0.000008 ; \mathrm{P} 1, p<$ 0.01; P2, $p<0.0003$; P3, $p<0.04$; P4, $p<0.0004$; P5, $p<0.02$; and P6, $p<0.002$. Comparisons of row $\mathrm{C}$ between CxNR1KO and control cortices revealed significant differences for all ages except for P1, P2, and $\mathrm{P} 3$, probably because of significant contraction in row $\mathrm{C}$ area at these early ages. $p$ values for other ages are as follows: $\mathrm{P} 0, p<0.0001 ; \mathrm{P} 4, p<0.001$; $\mathrm{P} 5, p<0.02$; and $\mathrm{P} 6, p<0.001$.

with our current findings. On the basis of the functional properties of barrel cortex neurons, Fox et al. (1996) inferred that topographic refinement of TCAs might be perturbed or intracortical connectivity might be altered. Our most recent findings in the CxNR1KO mice show that both TCA terminal fields and dendritic differentiation and orientation of barrel cortex cells are compromised (Datwani et al., 2002). Finally, in the barrel cortex, GABAergic elements also show patterns and alterations after peripheral lesions during the critical period (Lin et al., 1985; Akhtar and Land, 1991; Kiser et al., 1998). Thus, residual NMDAR function in the GABAergic barrel-field neurons might contribute to the TCA plasticity observed in the CxNR1KO mice.

TCA plasticity in CxNR1KO mice provides insights into the instructive role of neural activity. We propose that critical-period plasticity and its duration are set by the levels of afferent activity that start in the sensory periphery and propagate to the barrel cortex via the subcortical trigeminal centers. Lesion-induced alterations in TCA patterning may be a simple transfer of these effects from the brainstem to the thalamus and consequently to the S1 cortex. This may explain the recovery of barrel patterns after nerve lesion and cortical NMDAR blockade (Boylan et al., 2001). In this study, nerve lesions were placed after the brainstem patterns have already formed and at the onset of patterning in the VPM nucleus of the thalamus. Thus, the afferents projecting from one station to the next may already possess the necessary patterning information to enable their appropriate clustering.

In CxNR1KO mice, barrels as cellular modules do not form, and although TCAs representing the large whiskers on the snout segregate and develop patterns, these patterns are smaller than those seen in normal mice. It is noteworthy that the anterior sinus hair representation was generally absent in $\mathrm{CxNR} 1 \mathrm{KO} \mathrm{S} 1$ cortex, suggesting that relative levels of activity transmitted by different size vibrissas may be critical in determining the amount of cortical territory occupied by each whisker-related afferent. A recent study in the developing visual system indicates that relative levels of activity between competing sets of inputs are crucial for activity-dependent refinement of sensory connections. Stellwagen and Shatz (2002) showed that when the afferent input from one eye is made more active by use of agents that elevate cAMP, corresponding eye-specific layers in the lateral geniculate nucleus enlarge. If activity levels in both eyes are elevated, then eyespecific lamination develops normally. These findings provide strong support for the idea that the relative levels of activity rather than absolute levels are critical in regulating the amounts of target territories claimed by competing sets of afferents. A similar type of differential neural activity along elements representing large versus small whiskers and damaged versus intact whiskers may underlie the patterning and plasticity of TCAs in the barrel cortex, independent of NMDAR function in excitatory postsynaptic cells.

In conclusion, region-specific genetic perturbations of the NMDARs allow us to differentiate between the presynaptic and postsynaptic elements in patterning of the mammalian sensory cortex. Similar approaches for other receptor systems of glutamatergic transmission would allow dissection of their role in cortical patterning. Likewise, region-specific gene deletions in subcortical sensory pathways will yield important information about the role of a variety of gene products in pattern formation and neural plasticity and molecular switches that control the duration of critical periods.

\section{REFERENCES}

Akhtar ND, Land PW (1991) Activity-dependent regulation of glutamic acid decarboxylase in the rat barrel cortex: effects of neonatal versus adult sensory deprivation. J Comp Neurol 307:200-213.

Boylan CB, Kesterson KL, Bennett-Clarke CA, Chiaia NL, Rhoades RW (2001) Neither peripheral nerve input nor cortical NMDAR activity are necessary for recovery of a disrupted barrel pattern in rat somatosensory cortex. Brain Res Dev Brain Res 129:95-106.

Cooper NG, Steindler DA (1986) Monoclonal antibody to glial fibrillary 
acidic protein reveals a parcellation of individual barrels in the early postnatal mouse somatosensory cortex. Brain Res 380:341-348.

Cramer KS, Sur M (1995) Activity-dependent remodeling of connections in the mammalian visual system. Curr Opin Neurobiol 5:106-111.

Datwani A, Iwasato T, Itohara S, Erzurumlu RS (2002) NMDA receptor-dependent pattern transfer from afferents to postsynaptic cells and dendritic differentiation in the barrel cortex. Mol Cell Neurosci, in press.

Erzurumlu RS, Kind PC (2001) Neural activity: sculptor of "barrels" in the neocortex. Trends Neurosci 24:589-595.

Fox K, Schlaggar BL, Glazewski S, O'Leary DDM (1996) Glutamate receptor blockade at cortical synapses disrupts development of thalamocortical and columnar organization in somatosensory cortex. Proc Natl Acad Sci USA 93:5584-5589.

Goodman CS, Shatz CJ (1993) Developmental mechanisms that generate precise patterns of neuronal connectivity. Cell 72:77-98.

Iwasato T, Datwani A, Wolf AM, Nishiyama H, Taguchi Y, Tonegawa S, Knopfel T, Erzurumlu RS, Itohara S (2000) Cortex-restricted disruption of NMDAR1 impairs neuronal patterns in the barrel cortex. Nature 406:726-731.

Jhaveri S, Erzurumlu RS, Crossin K (1991) Barrel construction in rodent neocortex: role of thalamic afferents versus extracellular matrix molecules. Proc Natl Acad Sci USA 88:4489-4493.

Katz LC, Shatz CJ (1996) Synaptic activity and the construction of cortical circuits. Science 274:1133-1138.

Killackey HP (1973) Anatomical evidence for cortical subdivisions based on vertically discrete thalamic projections from the ventral posterior nucleus to cortical barrels in the rat. Brain Res 51:326-331.

Kiser PJ, Cooper NG, Mower GD (1998) Expression of two forms of glutamic acid decarboxylase (GAD67 and GAD65) during postnatal development of rat somatosensory barrel cortex. J Comp Neurol 402:62-74.
Lebedev MA, Mirabella G, Erchova I, Diamond ME (2000) Experiencedependent plasticity of rat barrel cortex: redistribution of activity across barrel-columns. Cereb Cortex 10:23-31.

Lebrand C, Cases O, Adelbrecht C, Doye A, Alvarez C, El Mastikawy S, Seif I, Gaspar P (1996) Transient uptake and storage of serotonin in developing thalamic neurons. Neuron 17:823-835.

Lebrand C, Cases O, Wehrle R, Blakely RD, Edwards RH, Gaspar P (1998) Transient developmental expression of monoamine transporters in the rodent forebrain. J Comp Neurol 401:506-524.

Lin CS, Lu SM, Schmechel DE (1985) Glutamic acid decarboxylase immunoreactivity in layer IV of barrel cortex of rat and mouse. J Neurosci 5:1934-1939.

Pallas SL (2001) Intrinsic and extrinsic factors that shape neocortical specification. Trends Neurosci 24:417-423.

Schlaggar BL, Fox K, O'Leary DD (1993) Postsynaptic control of plasticity in developing somatosensory cortex. Nature 364:623-626.

Steindler DA, O'Brien TF, Laywell E, Harrington K, Faissner A, Schachner M (1990) Boundaries during normal and abnormal brain development: in vivo and in vitro studies of glia and glycoconjugates. Exp Neurol 109:35-56.

Stellwagen D, Shatz CJ (2002) An instructive role for retinal waves in the development of retinogeniculate connectivity. Neuron 33:357-367.

Wallace H, Glazewski S, Liming K, Fox K (2001) The role of cortical activity in experience-dependent potentiation and depression of sensory responses in rat barrel cortex. J Neurosci 21:3881-3894.

Woolsey TA (1990) Peripheral alterations and somatosensory development. In: Development of sensory systems in mammals (Coleman EJ, ed), p 461. New York: Wiley.

Woolsey TA, Van der Loos H (1970) The structural organization of layer IV in the somatosensory region (S1) of mouse cerebral cortex: the description of a cortical field composed of discrete cytoarchitectonic units. Brain Res 17:205-242. 